\title{
A Fractal Ultra-High-Speed Oscillator/Distributor Network with Structural Robustness to Voltage and Temperature Gradients
}

\author{
Won-yong Choi, Sung-jin Kim, Kang-in Heo and Gyu Moon \\ VLSI Lab, Department of Electronic Engineering, Hallym University, \\ Chuncheon, Gangwon-do, Republic of Korea 200-702 \\ gmoon@hallym.ac.kr
}

\begin{abstract}
A study of a fractal structure of a clock generator and distributor is performed with possible use in today's ultra-high-speed, GHz range or higher, integrated circuit design and other future digital system applications. A fractal cell is composed of a simple ring oscillator with three inverting amplifiers, configuring a triangle shape, with an inverting amplifier placed at each side. Each side is then shared with an adjacent cell, which makes this fractal structure that is, theoretically, spreadable infinitely in $2 D$ domain. This fractal structure is proved to have insensitivities to supply voltage and temperature variations: less than $1 \%$ of clocks skew of a clock period, with given 3\% of supply voltage or $5 \%$ of temperature fluctuations. This is because any local disturbances or gradients will instantly and evenly be distributed to the whole networks yielding a global averaged state change. SPICE simulations are done with $0.5 \mu \mathrm{m}, 3 \mathrm{~V}, \mathrm{~N}$-Well CMOS process technology in order to prove the validity of the idea. A simple fractal CMOS layout, with 108 inverters, is also performed and included for future chip measurement.
\end{abstract}

Keywords: Fractal Structure, GHz Oscillator, Clock Skew, Jitter, Clock Generation and Distributtion, Process/Voltage/Temperature (PVT) Gradient

\section{Introduction}

Clock generation and distribution technique, in a few GHz-level or higher, for today's SOC and future ULSI chips is emerging as one of engineering's important bottlenecks, especially in terms of the overall performance of the whole digital system [1-3]. It is because the timing windows for recent digital systems have been scaling down to tens of a pico-second level during which data should be fetched and also processed, imposing a heavy timing constraint over a system data flow synchronization $[2,4-8]$.

The most popular and currently widely-used solution for an ultra-high-speed synchronization is Phase Locked-Loop (PLL) [9-12]. Along with symmetric clock line layout schemes, the PLL is known to provide synchronized system clocks with minimum level of clock skew, or jitters. However, with the physical nature of the clock line material characteristics, the jitters are still a limiting factor of systems overall performance, causing synchronization malfunctions even with today's state of the art PLLs $[2,8,13$ $15]$.

Several researches for controlling the clock jitters, or skew, have been focused to minimize the skew, and several different techniques are still undergoing review for actual on-system use. These techniques seem to have complex structures with a heavy routing area or clock signal distribution length limit [2, 8, 13, 16-19].

In this paper, we are proposing a novel way of $\mathrm{GHz}$ clock generation and distribution technique [20]. This technique, called Fractal Cellular Oscillator Network, adopts a fractal structure of a very simple oscillator, which can spread infinitely in $2 \mathrm{D}$ in the current 
silicon-based IC systems. Due to this inherent fractal structure, it minimizes the jitters caused by local gradients, yielding global averaging and thus minimized jitter signals for each and every node instantly. This means that even with voltage and/or temperature gradient, this technique can still generate clock signals within a very small jitter range.

Temporal or steady-state temperature and/or power supply gradients are given for the proposed technique's robustness proof.

\section{Structure of Fractal Cellular Oscillator Networks}

Figure 1 shows the structure of the fractal Cellular Oscillator Network (CON). As seen, the CON has a fractal modular structure, theoretically spreadable infinitely. Each branch is composed of an inverting amplifier, performing an inverting function.

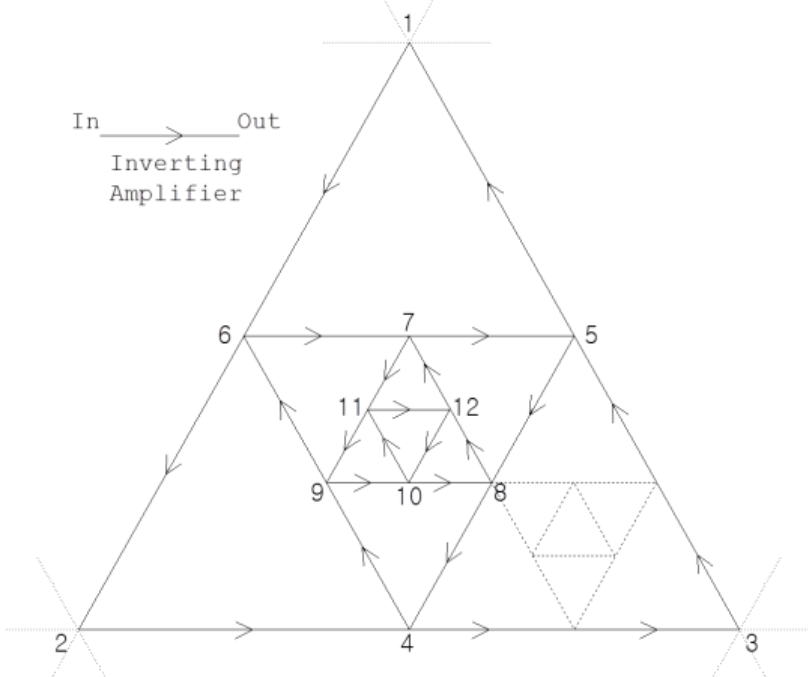

Figure 1. Structure of Fractal Cellular Oscillator Network

Here in this work, each branch is implemented with a CMOS inverter, as the simplest form, and each loop consists of an odd number of inverting nodes, which act as a type of a ring oscillator. Notice here that not just an inverter but any type of inverting amplifiers can be placed at each branch. Sharing each branch with adjacent three loop branches, this basic ring oscillator becomes a fractal cell of the fractal cellular oscillator network $(\mathrm{CON})$.

In this scheme in Figure 1, if we use the exact same circuitry for each branch, this CON generates three different oscillating signals from three different nodes, with $2 / 3 \pi$ phase shift among them $[6,8,15,20]$. These oscillating signals will have exact $50 \%$ of duty cycle, which is another important criterion in today's telecommunication digital systems $[8,14,15]$.

Once an external power supply is given for the CON, each loop will naturally and inherently oscillate with the exact same frequency. And even with a frequency change or a phase shift in one or several nodes in the network with voltage or temperature gradient, this local disturbance will spread out to the entire network, yielding an instant global state change with minimum skews among clock signals $[6,20]$. 


\section{CMOS Oscillator Network with an Inverter for Each Branch}

Figure 2 shows a simple example of a fractal CMOS cellular oscillator network that is made up of only 108 inverters.

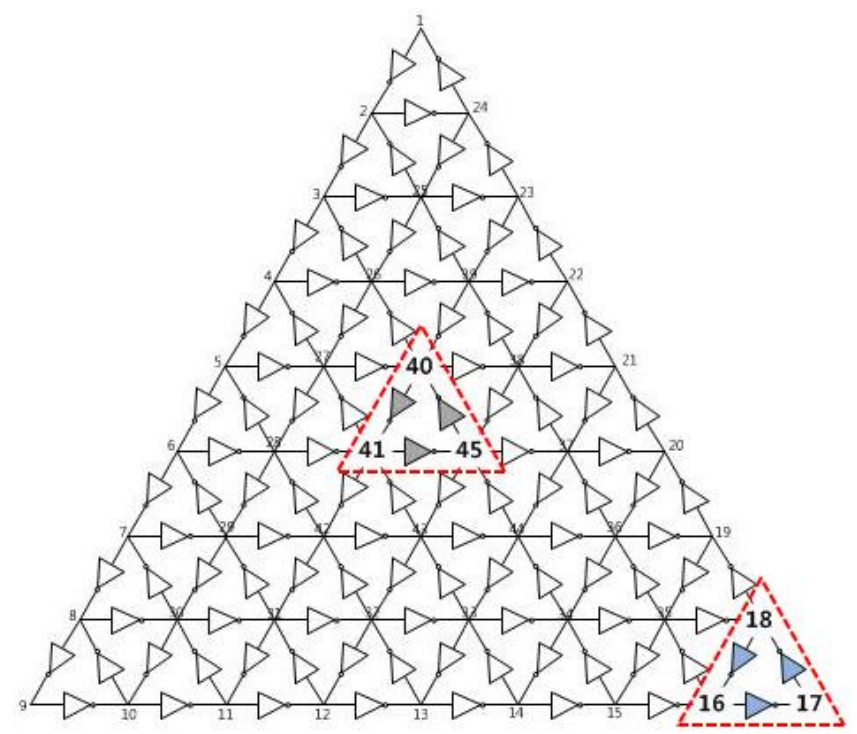

Figure 2. Fractal CMOS Cellular Oscillator Network With 108 Inverters

A way of implementing the CON is hereby suggested and displayed in Figure 3 using today's multilayer integrated circuit process and package technique. Analog and digital planes are separated for minimum interference between two different planes, and in the middle the proposed CON can be installed as shown.

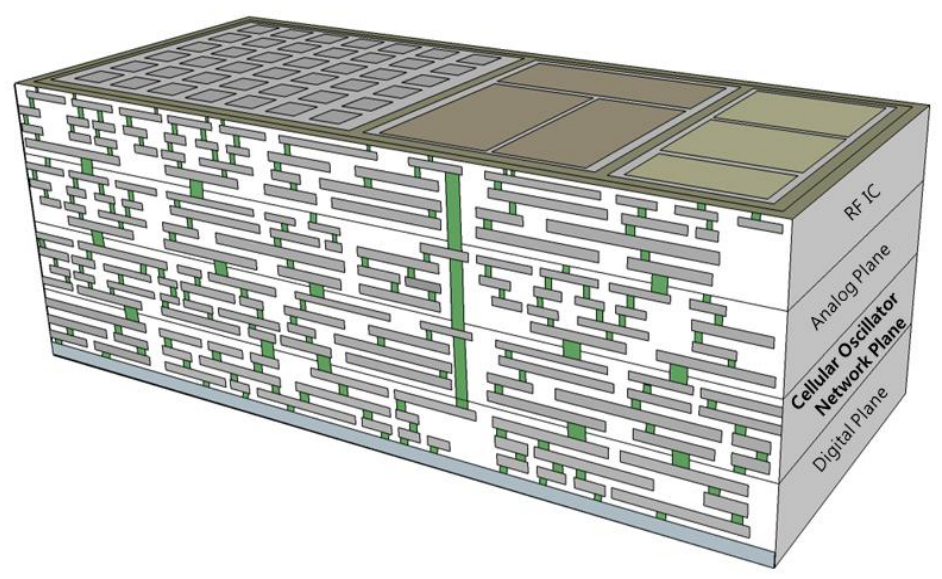

Figure 3. The CON Silicon Implementation in Multilayer CMOS/ Multi-Chip Module Package Technique

Notice in this scheme shown in Figure 1, there will be an instant global clock signal distribution with unbalanced load conditions, and in this paper, even with local power supply and/or temperature disturbances. The CON will have an inherent robustness against these different kinds of temporal or static gradients, with possibly minimum skews. 


\section{Clock Skew Simulation Results of Fractal Cellular Oscillator Network}

Simulation results of the Figure 2 in time domain are shown in Figure 4.

The temperature and power supply are the same for the whole circuit with $300 \mathrm{~K}$ and 3 volts, respectively. As seen here, only three different oscillating signals are detected from the network, with a 120-degree phase difference among them.

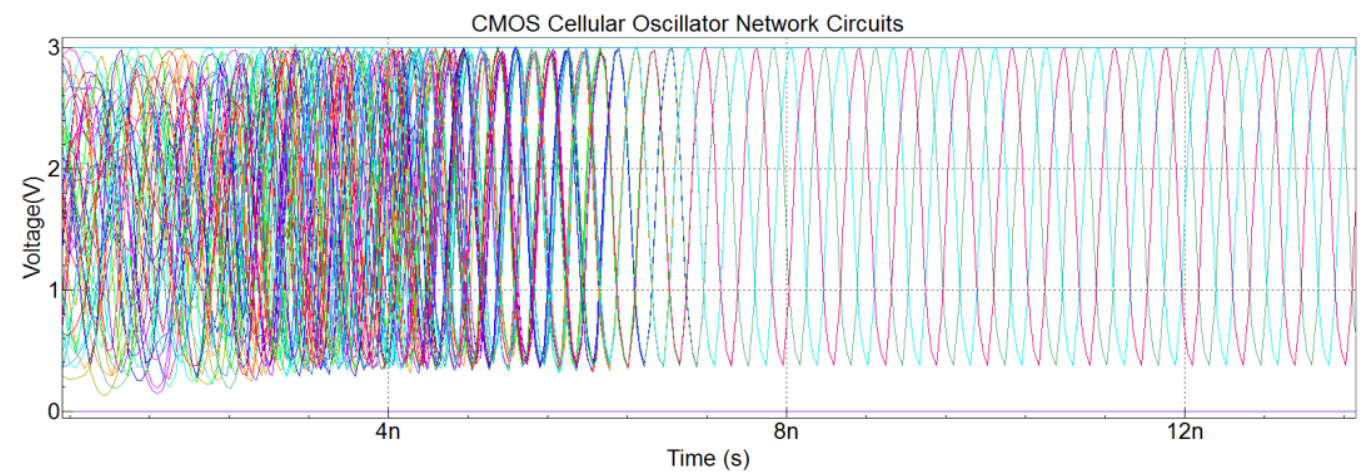

Figure 4. Simulation Results for the Figure 2

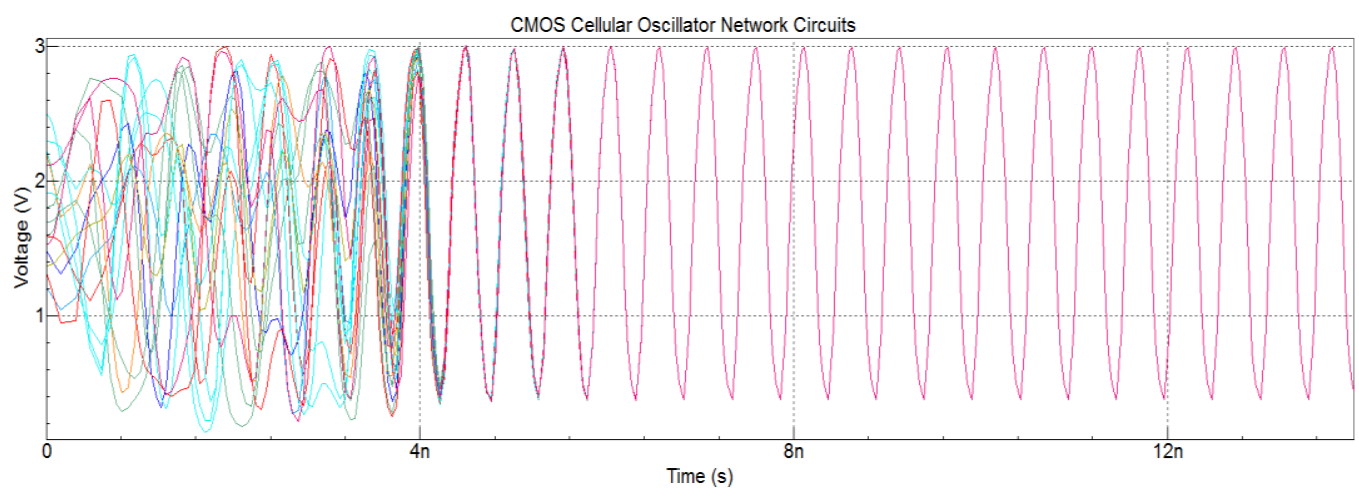

Figure 5. Fifteen In-Phase Nodes Simulation Results

In Figure 5, only 15 in-phase nodes signals in Figure 2 are displayed separately. With this fractal structure, and with feedback loops in the networks, fifteen nodes generate exact same signals.

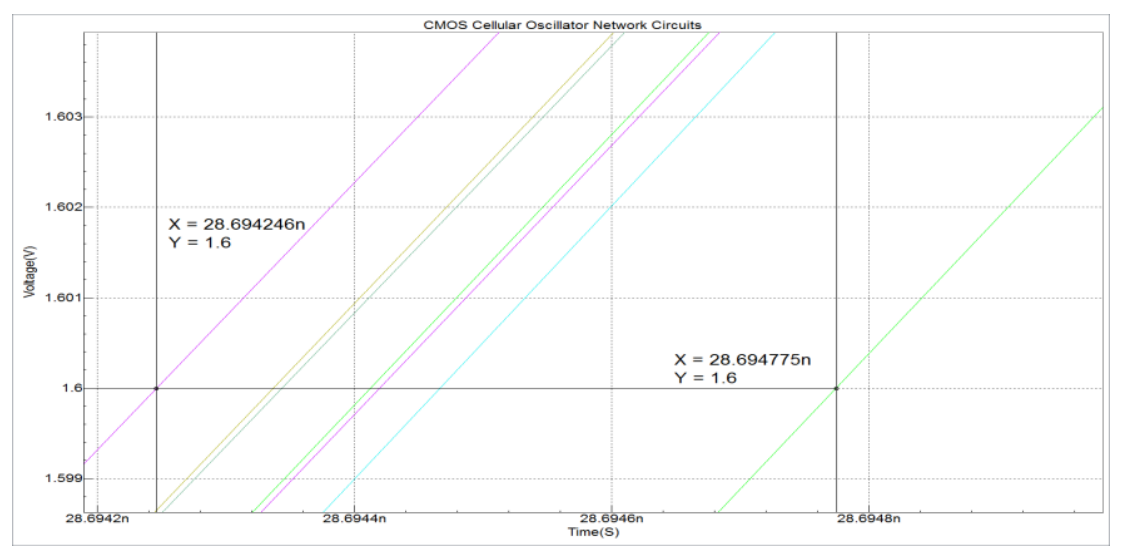

Figure 6. In-phase Nodes Clock Signals Skew Measurement 


\section{Simulation Results with Power Supply Voltage/Temperature Gradients}

Simulations were done with CMOS n-well technology, along with $3 \mathrm{~V}, 0.5 \mathrm{um}$ minimum feather size (both $\mathrm{N}$ - and P-MOS) conditions. Supply voltage is assumed changing $3 \%$ range, while the temperature is fluctuating within $5 \%$ of a given ambient temperature, a room temperature $300 \mathrm{~K}$.

\subsection{Clock Skew Simulation with Supply Voltage Gradient}

Figure 7 shows clock skew simulation measurements when power supply is changing locally up to 2,5 , and $8 \%$, respectively. The (a) is when the local change is given only in the center loop (node 40, 41, 45 in Figure 2) and the (b) is for the corner loop (node 16, 17, 18 in Figure 2).

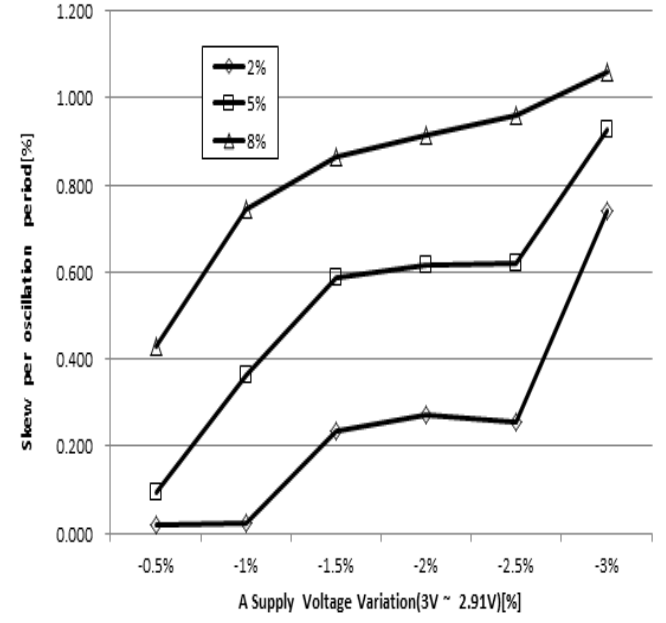

(A) Center Loop and

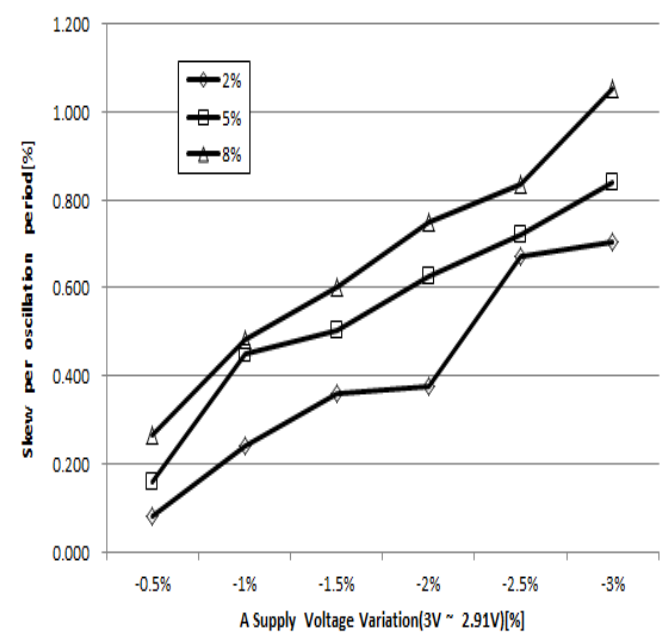

(B) Corner Loop

Figure 7. Clock Skews with Local Power Supply Voltage Change

As read from the Table 1, maximum of $\sim 1 \%$ of clock period is measured among inphase nodes with $8 \%$ of supply voltage is given. The insensitivities for a local supply voltage fluctuation is clearly witnessed.

Table 1. Skew Variation Results for a Local Supply Voltage Change (Center and Corner)

\begin{tabular}{|c|c|c|c|c|c|c|c|c|}
\hline \multicolumn{9}{|c|}{ The Center of Networks at Supply Voltage Variation } \\
\hline \multicolumn{3}{|c|}{$\begin{array}{c}\text { Volt } \\
\text { Variation } \\
\end{array}$} & $-0.5 \%$ & $-1 \%$ & $-1.5 \%$ & $-2 \%$ & $-2.5 \%$ & $-3 \%$ \\
\hline \multirow{3}{*}{$\begin{array}{c}\text { Skew } \\
\text { Rate[\%] }\end{array}$} & \multirow{3}{*}{$\begin{array}{l}\text { Cell } \\
\text { Area }\end{array}$} & $2 \%$ & 0.021 & 0.023 & 0.235 & 0.273 & 0.257 & 0.742 \\
\hline & & $5 \%$ & 0.095 & 0.364 & 0.587 & 0.618 & 0.620 & 0.929 \\
\hline & & $8 \%$ & 0.430 & 0.745 & 0.866 & 0.916 & 0.960 & 1.061 \\
\hline \multicolumn{9}{|c|}{ Supply Voltage Range : $3 \sim 2.91 \mathrm{~V}$} \\
\hline \multicolumn{9}{|c|}{ The Corner of Networks at Supply Voltage Variation } \\
\hline \multicolumn{3}{|c|}{$\begin{array}{c}\text { Volt } \\
\text { Variation }\end{array}$} & $-0.5 \%$ & $-1 \%$ & $-1.5 \%$ & $-2 \%$ & $-2.5 \%$ & $-3 \%$ \\
\hline \multirow{3}{*}{$\begin{array}{c}\text { Skew } \\
\text { Rate[\%] }\end{array}$} & \multirow{3}{*}{$\begin{array}{l}\text { Cell } \\
\text { Area }\end{array}$} & $2 \%$ & 0.081 & 0.240 & 0.361 & 0.375 & 0.672 & 0.703 \\
\hline & & $5 \%$ & 0.159 & 0.451 & 0.502 & 0.628 & 0.722 & 0.841 \\
\hline & & $8 \%$ & 0.265 & 0.483 & 0.602 & 0.748 & 0.836 & 1.054 \\
\hline
\end{tabular}




\subsection{Clock Skew Simulation with Temperature Gradient}

Local temperature change is given, from $0 \%$ up to $100 \%$ for the whole nodes, and the skew was measured for each case. Figure 8 shows the simulation results.

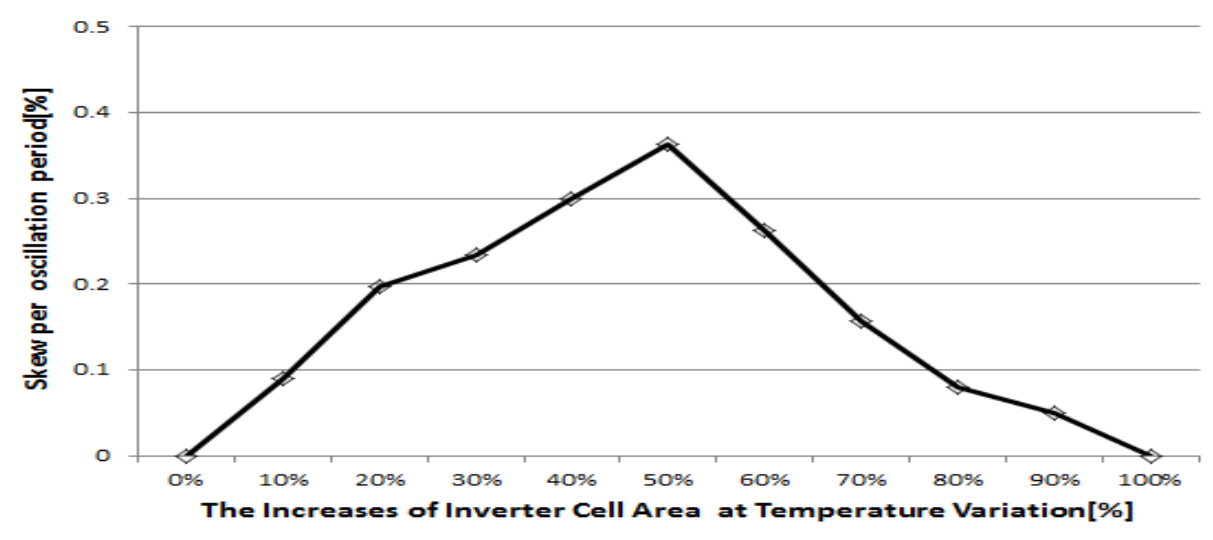

Figure 8. Clock Skew Variation with a Different Local Node Numbers (\%)

As seen, the more nodes (cells) have temperature changes, the more skew is measured, at $50 \%$ the largest of $0.35 \%$.

Table 2. Skew Variations for Maximum 5\% of Temperature Change

\begin{tabular}{|c|c|c|c|c|c|c|c|}
\hline $\begin{array}{c}\text { Temp } \\
\text { Variation }\end{array}$ & $-5 \%$ & $-3 \%$ & $-1 \%$ & $0 \%$ & $1 \%$ & $3 \%$ & $5 \%$ \\
\hline $\begin{array}{c}\text { Skew } \\
\text { Rate[\%] }\end{array}$ & 0.347 & 0.095 & 0.062 & 0 & 0.065 & 0.185 & 0.340 \\
\hline \multicolumn{7}{|c|}{ Operation Temperature Range: $-40 \sim 85^{\circ} \mathrm{C}$} \\
\hline
\end{tabular}

\section{CMOS Layout}

Figure 9 is a CMOS layout of 108 inverters CON shown in Figure 2. 


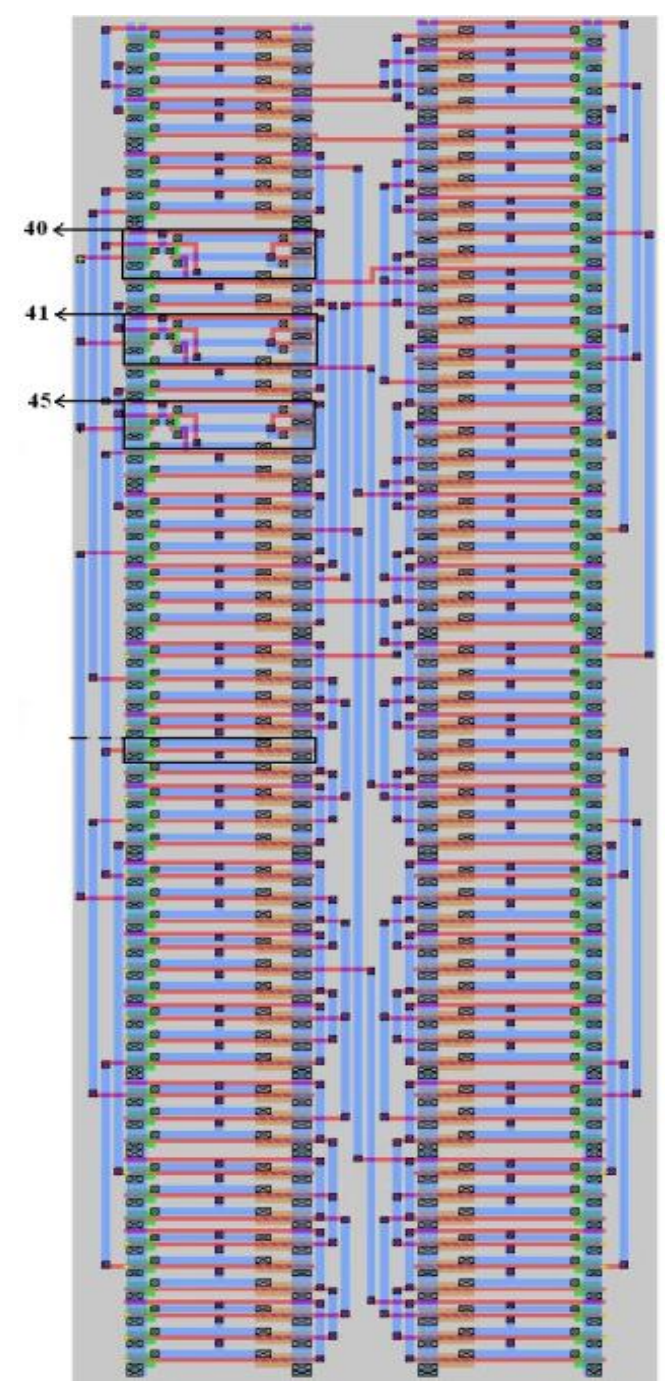

Figure 9. CMOS Layout of Figure 2

\section{Conclusions}

A primitive idea of fractal ultra-high-speed oscillator and distributor network is newly presented. Using an idea from well-known fractal model, this new and very simple approach of clock generation and distribution technique is tested and proved to work well in $\mathrm{GHz}$ range with minimum skews. With supply voltage and temperature gradients, simulations were performed to measure clock skews in different cases. With its inherent fractal structure, the cellular oscillator network is proven to have less than $1 \%$ and $0.4 \%$ clock skews of a clock period with given $3 \%$ of power supply and 5\% of temperature fluctuations, respectively. This implies that the fractal cellular oscillator network can be adopted in $\mathrm{GHz}$ clock generation and distribution in a single chip, and chip-to-chip and system-to-system (SOC) applications as well. With its simple structure, and easy-to-implementation, the proposed technique is expected to be adopted in future digital systems. Layout is done and a chip is under fabrication for a future test.

\section{Acknowledgements}

This research was supported by Hallym University Research Fund, 2012(HRF 201206 - 006). This work was also supported by IDEC(EDA Tool) in Korea. 


\section{References}

[1] Q. Zhu and S. Tam, "Package Clock Distribution Design Optimization for High-speed and Low Power VLSI's", IEEE Trans. on Components, Packaging and Manufacturing Technology, Part B: Advanced Packaging, vol. 20, no. 1, (1997), pp. 56-63.

[2] E. G. Friedman, "Clock Distribution Networks in Synchronous Digital Integrated Circuits", Proceedings of the IEEE, vol. 89, no. 5, (2001), pp. 665-692.

[3] L. Zhang, A. Carpenter, B. Ciftcioglu, A. Garg, M. Huang and H. Wu, "Injection-locked Clocking: A Low-power Clock Distribution Scheme for High-performance Microprocessors", IEEE Trans. on Very Large Scale Integration(VLSI) Systems, vol. 16, no. 9, (2008), pp. 1251-1256.

[4] G. Geannopoulos and X. Dai, "An Adaptive Digital Deskewing Circuit for Clock Distribution Networks", Proceeding of the IEEE 1998 International Solid-State Circuits Conference(ISSCC) Digest of Technical Papers, San Francisco, USA, (1998) February 5-7.

[5] H. Mizuno and K. Ishibashi, "A Noise Immune GHz Clock Distribution Scheme using Synchronous Distributed Oscillators", Proceeding of the IEEE 1998 International Solid-State Circuits Conference(ISSCC) Digest of Technical Papers, San Francisco, USA, (1998) February 5-7.

[6] S.-K. Hwang and G. Moon, "A Ultra High Speed Clock Distribution Technique using a Cellular Oscillator Network", Proceedings of the IEEE 2000 International Symposium on Circuits and Systems(ISCAS), Geneva, Switzerland.

[7] C.-C. Chung and C.-Y. Lee, "An All-digital Phase Locked Loop for High Speed Clock Generation", IEEE Journal of Solid-State Circuits, vol. 38, no. 2, (2003), pp. 347-351.

[8] A. Hajimiri and T. H. Lee, "The Design of Low Noise Oscillators", Kluwer Academic Publishers, Norwell, USA, (2003).

[9] L. Li, L. Tee and P. R. Gary, "A 1.4 GHz Differential Low-noise CMOS Frequency Synthesizer using a Wideband PLL Architecture", Proceeding of the IEEE 2000 International Solid-State Circuits Conference(ISSCC) Digest of Technical Papers, San Francisco, USA, (2000) February 7-9.

[10] V. Gutnik and A. P. Chandrakasan, "Active GHz Clock Network using Distributed PLLs", IEEE Journal of Solid-State Circuits, vol. 35, no. 11, (2000), pp. 1553-1560.

[11] H.-H. Hsieh, C.-T. Lu and L.-H. Lu, "A 0.5-V 1.9-GHz Low-power Phase Locked Loop in 0.18- $\mu \mathrm{m}$ CMOS", Proceedings of the IEEE 2007 International Symposium on VLSI Circuits(VLSIC), Kyoto, Japan, (2007) June 14-16.

[12] J.-W. Moon, K.-C. Choi and W.-Y. Choi, "A 0.4-V, 90 350-MHz PLL with an Active Loop-Filter Charge Pump", IEEE Trans. on Circuit and Systems II: Express Briefs, vol. 61, no. 4, (2014), pp. 319323.

[13] A. Hajimiri, S. Limotyrakis and T. H. Lee, "Jitter and Phase Noise of Ring Oscillators", IEEE Journal of Solid-State Circuits, vol. 34, no. 6, (1999), pp. 790-804.

[14] N. H. E. Weste and D. M. Harris, "CMOS VLSI DESIGN(A Circuits and Systems Perspective)", Addison Wesley, USA, (2010).

[15] R. Jacob Baker, "CMOS(Circuit Design, Layout, and Simulation)", John Wiley \& Sons, Inc., IEEE Press, USA, (2010).

[16] S. C. Chan, K. L. Shepard and P. J. Restle, "1.1 to 1.6GHz Distributed Differential Oscillator Global Clock Network", Proceeding of the IEEE 2005 International Solid-State Circuits Conference(ISSCC) Digest of Technical Papers, San Francisco, USA, (2005) February 10.

[17] J. Rosenfeld and E. G. Friedman, "Design Methodology for Global Resonant H-Tree Clock Distribution Networks", IEEE Trans. on Very Large Scale Integration(VLSI) Systems, vol. 15, no. 2, (2007), pp. 135148.

[18] J. Minz, X. Zhao and S.-K. Lim, "Buffered Clock Tree Synthesis for 3D ICs under Thermal Variations", Proceeding of the 13th Asia and South Pacific Design Automation Conference (ASPDAC'08), Seoul, Republic of Korea, (2008) March 21-24.

[19] V. F. Pavlidis, I. Savidis and E. G. Friedman, "Clock Distribution Networks in 3-D Integrated System", IEEE Trans. on Very Large Scale Integration(VLSI) Systems, vol. 19, no. 12, (2011), pp. 2256-2266.

[20] W.-Y. Choi, S.-J. Kim, K.-In Heo and G. Moon, "Design of CMOS GHz Cellular Oscillator/Distributor Network with Supply Voltage and Ambient Temperature Insensitivities", Proceeding of the International Workshop on Ubiquitous Science and Engineering 2015 Advanced Science and Technology Letters(ASTL), Jeju, Republic of Korea, (2015) April 15-18. 


\section{Authors}

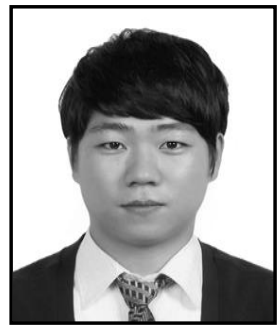

Wonyong Choi was born in Chuncheon, Korea, in 1986. He received the B.S. degree in Electronic Engineering in 2013, and is currently working toward the M.S. degree in Electronic Engineering from Hallym University, Chuncheon, South Korea. His research interests are the design of analog-mixed signal circuits, MEMS, Microsystems, and CMOS sensors and applications.

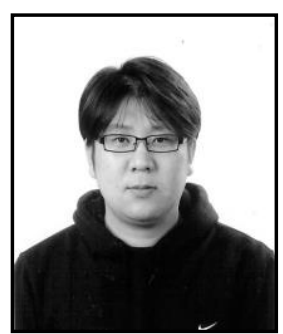

Sungjin Kim was born in Gapyeong, Korea, in 1987. He received the B.S. degree in Electronic Engineering from Hallym University, Chuncheon, South Korea in 2014. He is currently working toward the M.S. degree at the same university. His major research interests are the design of analog-mixed signal circuits, PLLs, and bionics.

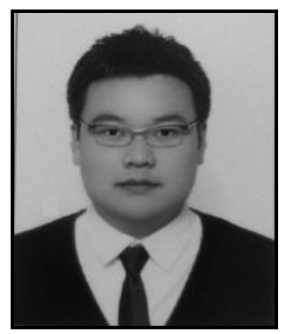

Kangin Heo was born in Goyang, Korea, in 1986. He received the B.S. degree in Electronic Engineering from Hallym University, Chuncheon, South Korea in 2014. He is currently working toward the M.S. degree at the same university and his current research interests are high speed VLSI design, low-power circuits and DLLs.

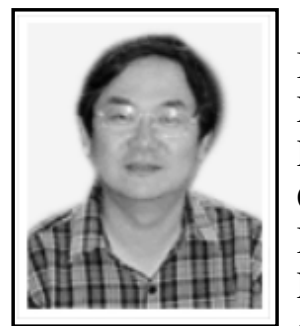

Gyu Moon was born in Daegu, Korea, in 1959. He received the B.S. degree in Control and Instrumentation Engineering from Seoul National University, Seoul, South Korea in 1982, and the M.S. and $\mathrm{Ph} . \mathrm{D}$. degrees in the Department of Electrical Engineering and Computer Science at the George Washington University, Washington D.C., USA in 1990 and 1993. From 1982 to 1988 , he worked for the Electronics and Telecommunication Research Institute (ETRI), Korea, as a Member of Technical Staff. His current interests are Circuits and Systems, Mixed- mode IC Design, Digital Security Systems, and ASIC. 
International Journal of Control and Automation Vol. 8, No. 6 (2015) 